\title{
Review
}

\section{Decision-Making in Diagnosis and Management of Extraintestinal Manifestations of Inflammatory Bowel Disease}

\author{
Nicholas V. Costrini, MD, PhD, MBA, AGA-F* \\ Borland Groover Clinic, PA, 4800 Belfort Road, Jacksonville, FL 32256, USA \\ "Corresponding author \\ Nicholas V. Costrini, MD, PhD, MBA, AGA-F \\ Borland Groover Clinic, PA, 4800 Belfort Road, Jacksonville, FL 32256, USA; Phone: 406-300-2524; Fax. 904-348-0830; E-mail: drcostn@gmail.com
}

\section{Article information}

Received: May $6^{\text {th }}, 2021$; Revised: June $5^{\text {th }}, 2021$; Accepted: June $7^{\text {th }}$, 2021; Published: June 19th 2021

\section{Cite this article}

Costrini NV. Decision-making in diagnosis and management of extraintestinal manifestations of inflammatory bowel disease. Gastro Open J. 202I; 5(I): 4-I2. doi: $10.17 / 40 / G O J-5-133$

\section{| ABSTRACT |}

In the absence of certainty regarding the causes of both inflammatory bowel disease (IBD) and its extraintestinal manifestations (EIMs), there is necessarily ambiguity in both academic and clinical arenas concerning the diagnosis, classifications, and treatments of EIMs. While the "true" EIMs are considered extensions of the IBD gut pathogenesis with an immunologically mediated inflammatory consequence, other EIMs are considered to be complications of IBD itself or its treatment. A third group of IBD EIMs includes those disorders which seem to occur more often in IBD but for which an etiologic or pathophysiologic connection to IBD is highly theoretical. Patients with IBD and EIMs tend to have more severe, long-duration disease, and a reduced quality of life. EIMs presentation may or may not parallel IBD gut inflammatory activity. The clinical decision-making processes necessary for successfully managing simultaneously the gut component of IBD and its EIMs are presented. Based upon clinical experience and review of leading publications, the consensus of best practices, differential diagnoses for EIMs, and current management programs are presented with enumeration of specific decisions and considerations required for successful management of EIMs. EIMs of inflammatory bowel disease reflect the immunopathologic common ground and hence the systemic nature of the IBD. A defined decision-making process is offered which includes consultations and attention to the differential diagnosis to avoid not uncommon mistakes in diagnosis. Management of all EIMs requires assessment of both the clinical and pathologic status of the gut component of IBD combined with judicious selection of general and /or immunosuppression therapy for the EIMs.

\section{Keywords}

Ulcerative colitis; Crohn's disease; Erythema nodosum; Pyoderma gangrenosum; Primary sclerosing cholangitis; Spondyloarthritis; Uveitis; Paradoxical dermatitis.

\section{INTRODUCTION}

W hile considerable progress has been made in determining the cause(s) of the principal inflammatory bowel diseases (IBD), crohn's disease (CD) and ulcerative colitis (UC), much uncertainty remains. The keys to understanding IBD revolve around a complex interplay of an altered microbiome, genetic predisposing factors, and environmental challenges. ${ }^{1-3}$ As research and clinical trials pertaining to the gastrointestinal diseases continue, in recent years, the extraintestinal manifestations (EIM) of IBD have gained increased attention. Efforts have centered upon explaining their pathogenesis, ${ }^{4-6}$ developing clinically useful classifications of the various EIMs, and determining the most appropriate treatment programs. EIMs may occur at any time before (20\% of patients) or after the diagnosis of IBD is established; and their prevalence increases with disease duration. The frequency of EIMs (up to $50 \%$, 7), the risks associated the EIMs (i.e., crippling arthritis, vision loss, cutaneous scarring, cancers, life-threatening venous thrombosis), and their impact on quality of life require that IBD be considered a systemic inflammatory disorder. In order to provide state of the art care, gastroenterologists are challenged to approach the diagnosis and management of both the gastrointestinal and extraintestinal manifestations of IBD with equal vigor. 


\section{DEFINITION, PATHOPHYSIOLOGY AND CLASSIFICATION OF EXTRAINTESTINAL MANIFESTATIONS}

EIMs are classified in restricted or universal patterns based upon an immunologically-informed or a disciplined interpretation of the term "extraintestinal." The European Crohn's and Colitis Organization (ECCO, 7) defines the EIMs of IBD (in this communication labeled as "true" EIMs) as follows: "An inflammatory pathology in a patient with IBD that is located outside the gut and for which the pathogenesis is either dependent on extension/ translocation of immune responses from the intestine, or is an independent inflammatory event perpetuated by IBD or that shares a common environmental or genetic predisposition with IBD." ECCO invokes the "mechanistic" definition of EIMs while admitting that most of the immunologic mechanisms proposed have yet to be proven. That notwithstanding, the EIMs of the skin, eye, bone, and hepatobiliary targets are accepted as those which are immunologically mediated as defined above, i.e., translocation of the immune response. ECCO and most clinicians recognize that IBD carries with it risks of non-inflammatory extraintestinal disorders that are secondary to the intestinal disease itself, secondary to the treatment of intestinal disease, or by observation seem to occur more often in association with IBD but through as yet very much undefined mechanisms. These add to the particularly clinically relevant, and more inclusive EIM classification scheme (Table 1) but offer the IBD clinician a daunting check list of extraintestinal disorders, which by any mechanism, require vigilance and care.

\section{PATHOGENESIS AND CLASSIFICATION OF "TRUE" EXTRAINTESTINAL MANIFESTATIONS}

The exact explanation(s) for the pathogenesis of CD and UC remain elusive. Hence, the same may be said for the pathogenesis of this class of IBD EIMs. For both IBD and the "true" EIMs, it is likely that genetic, environmental, immunologic, and microbiome factors interact, participate and modify gut inflammatory processes to orchestrate what is generically advanced as "loss of immune tolerance" leading to variably expressed gut inflammation. ${ }^{1-4,7}$ It is this complex pathogenesis which is extended to provoke inflammatory EIM events in skin, bone and joint, eye, and hepatobiliary systems. There are several compelling mechanisms proposed. ${ }^{7}$ EIMs may arise from an extension of antigen-specific immune responses from the intestine to non-intestinal sites. In this model, gut proteins and peptides serve as epitopes or immunologic determinants which can also be found in target EIM tissues. Epitopes recognized by activated lymphocytes, provoke cytokine release and the inflammatory response. Alternatively, the gut inflammatory events may extend to the skin, eyes, bones, and liver through circulating antibodies to shared gut and non-gut epitopes. ${ }^{8,9} \mathrm{It}$ is also possible that EIMs arise from an entirely independent inflammatory event influenced by a genetic predisposition and environmental factors.

Rather than having IBD serve as the centerpiece for the discussion of EIMs, it is prudent to look at the relationship between IBD and other inflammatory disorders as falling under the umbrella of immunologically-mediated inflammatory diseases (IMIDs).${ }^{10}$ This concept offers a theory that in-part explains why, for example, IBD occurs more often in the rheumatoid, spondyloarthritic, and psoriatic populations and why, conversely, these IMIDs occur more often in the IBD population than in the general population. The immunological mechanism underlying the IMIDs is depicted in the cartoon of Figure 1. In nearly all tissues of the body, the dendritic cells are those which monitor and respond to favorable and unfavorable elements in the local environment, i.e., the gut microbiome, antigens or epitopes via both blood and airways, etc. The immunologic response promotes the release of IL-23, the proinflammatory cytokine tissue necrosis factor-alpha (TNF- $\alpha$ ) and others that affect cellular targets. Such immunologic sequential events promote the panorama of IMIDs which may seem at first glance as totally unrelated to one another. The assembled classes IMIDs and the EIMs of IBD declare perhaps common pathways. Additionally, the undeniable impact of anti-TNF- $\alpha$ agents (i.e., in-

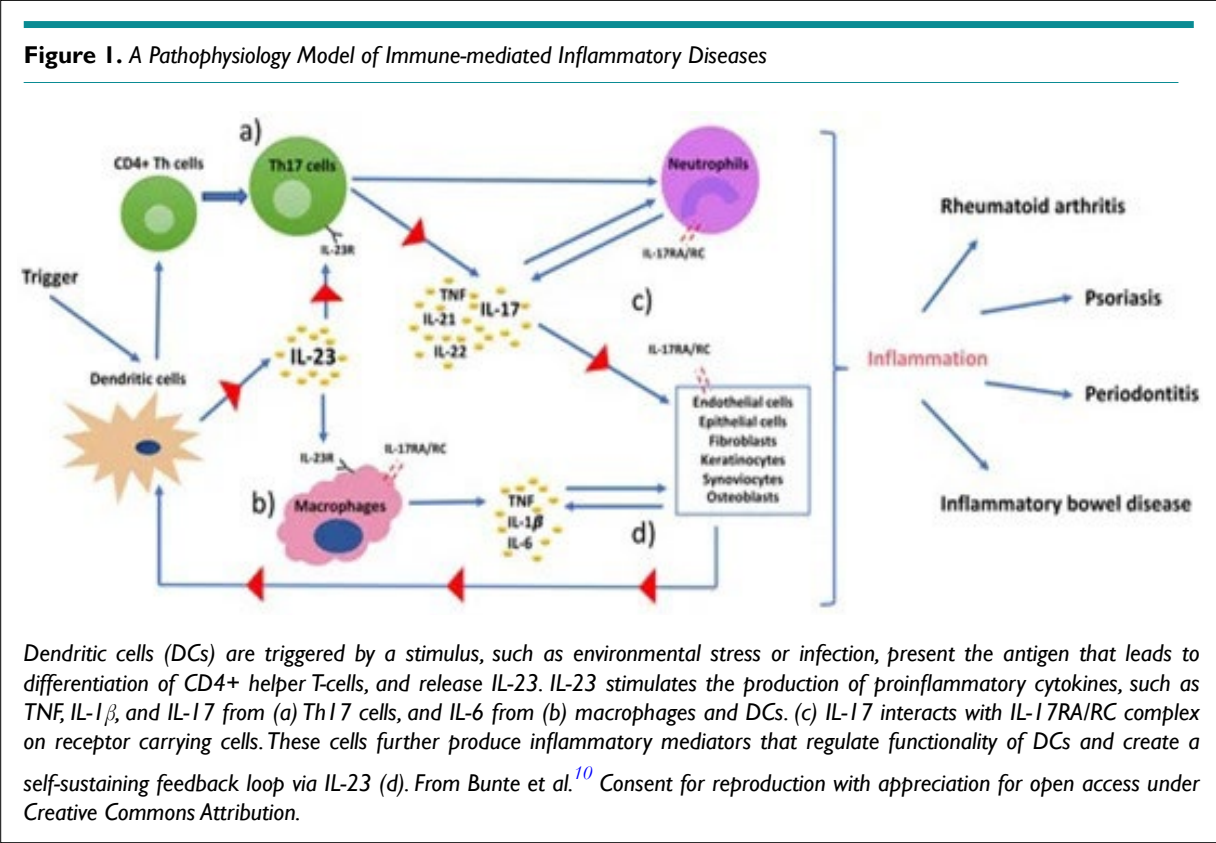


fliximab) and anti-IL-23 agents (i.e., ustekinumab) on both IBD, the various EIMs, and disorders that occur more often in the IBD population (group 3 in Table 1) lend support for this IMID theory.

\section{CLINICAL CHARACTERISTICS OF "TRUE" EIMS AND THE DECISIONS REQUIRED IN MANAGEMENT |}

The "true" EIMs as per ECCO are categorized according to clinical behavior relative to the activity of the gut component of IBD and the EIM response to anti-TNF- $\alpha$ biologics (Table 2).

\section{Mucocutaneous Extraintestinal Manifestations}

Aphthous stomatitis ulcers are among the most common ${ }^{11,12}$ of the EIMs, occur in CD (9.8-12.9\%) and UC (3.5-5.4\%) and are more often associated with active IBD. It usually appears as a mildly painful oral mucosa ulcer. It must be differentiated from a common viral herpetic ulcer. Excellent photographs of typical aphthous ulcers and other skin lesions of IBD may be found in Huang et al. ${ }^{9}$ When a patient with IBD presents with aphthous ulcers, several questions need to answered in making the important management decisions. These include the following:

1. Is the lesion an aphthous EIM lesion or opportunistic herpesvirus lesion? Consultation may be prudent.
2. Is the patient in remission? Occult, active disease may be present. ${ }^{13,14}$ While oral, topical therapy (viscous lidocaine, topical steroids) may provide relief, reassessment of the gut activity and perhaps that management program is in order.

3. Does the patient have active inflammatory gut disease defined variably by endoscopy, fecal calprotectin, C-reactive protein, and $\mathrm{CBC}$ ? Make every effort to stay with the current biologic or at least within class rather than switching to another agent. If alternative biologic therapy is planned, it is prudent to avoid the use of the principally gut-specific biologic, vedolizumab. While vedolizumab may ameliorate the oral lesions, it has been reported to exacerbate some EIMs. ${ }^{15}$

4. For recurrent, severe, or resistant oral disease, short-term, systemic steroids and consultation with an oral surgeon are in order. In such cases the anti-TNF- $\alpha$ biologic infliximab is very often the drug of choice.

\section{Erythema Nodosum}

This dermatological EIM of IBD occur with nearly equal prevalence or with a slight increase in $\mathrm{CD}(7.6 \% \text { vs. } 3.6 \% \text { in UC) })^{11}$ and offers a female predilection. Like other "true" EIMs erythema nodosum (EN) tends to arise during a flare or during a course of active IBD. Pathologically, these lesions reveal inflammatory lympho-histocytic infiltration of the lower dermis and subcutane-

\begin{tabular}{l}
\hline Table I. Classification of Extraintestinal Manifestations of Inflammatory Bowel Disease, $4,7,8$ \\
\hline I. “True" EIMs -Extensions of gut immunopathology \\
Mucocutaneous: oral/metastatic CD, erythema nodosum, pyoderma gangrenosum, Sweet's syndrome \\
Bone and Joint: spondyloarthritis - peripheral and axial \\
Eye: episcleritis, scleritis, uveitis \\
Hepatopancreaticobiliary: primary sclerosing cholangitis \\
2. Complications of inflammatory bowel disease or its treatment \\
Anemia, metabolic bone disease, vitamin deficiencies, portal vein thrombosis, non-alcoholic fatty liver \\
disease, cardiovascular and cerebrovascular disease, opportunistic infections, cataracts, "paradoxical” \\
inflammatory skin and joint disease due to anti-TNF agents, drug-induced liver disease, \\
nephritis, skin cancer, pancreatitis, peripheral neuropathy \\
3. Condition associated with IBD through unknown mechanisms \\
Non-inflammatory arthralgias, psoriasis, hidradenitis suppurativa, eczema, vitiligo, autoimmune \\
hepatitis, granulomatous hepatitis, autoimmune pancreatitis, type I diabetes, autoimmune thyroiditis. \\
\hline
\end{tabular}

\begin{tabular}{|c|c|c|c|c|}
\hline EIM & Parallel Course of IBD & Separate Course of IBD & May or May not Parallel & Response to Anti-TNF- $\alpha$ \\
\hline Axial arthropathy & & & & ++ \\
\hline Peripheral arthropathy & Y (type I) & Y(type 2) & & +++ \\
\hline Erythema nodosum & Y & & & ++ \\
\hline Pyoderma gangrenosum & & & Y & ++ \\
\hline Oral aphthous ulcers & Y & & & +++ \\
\hline Metastatic CD & & $Y$ & & ++ \\
\hline Sweet's Syndrome & & & $\mathrm{Y}$ & + \\
\hline Episcleritis & Y & & & +++ \\
\hline Scleritis & & $Y$ & & ++ \\
\hline Uveitis & & Y & & +++ \\
\hline Primary sclerosing cholangitis & & & Y & --- \\
\hline
\end{tabular}


ous fat, panniculitis. ${ }^{9}$ Thus, EN may present anywhere subcutaneous fat is present. Most often lesions occur on the lower limbs. Only rarely does EN antedate the diagnosis of IBD but rather occurs when the disease is well-established. The lesions are pink, non-ulcerating, painful, and may be single or multiple. Treatment is directed toward assessment and resolution of the active gut inflammation which will lead to improvement in both the intestinal and skin manifestation of IBD in most cases.

Table 1 reveals that many of the "true" EIMs follow the course of the gastrointestinal pathology. If the patient offers no clinical evidence of active intestinal disease, caution is required. ${ }^{13,14}$ Most reports of EIMs and the relationship to IBD gut activity have employed clinical measures of disease activity ${ }^{12}$ such as the Crohn's Disease Index of Severity. Clinical tools do not accurately define the level of activity as defined with endoscopic and microscopic assessments. Additionally, in the main, complete endoscopic remission is a minority position, typically $25-30 \%$, for the IBD population. Hence, a prudent course is to assess the pathologic status of gut activity in every IBD-EIM patient (i.e. fecal calprotectin, endoscopy, cross-sectional imaging, C-reactive protein) in addition to the apparent clinical status. Treating the clinically quiescent but pathologically active intestinal disease may be the very best means of treating the recalcitrant or recurrent EIM. ${ }^{16}$

The important clinical decisions to be made when caring for an IBD patient presenting with EN include:

1. Be certain of the diagnosis of EN. Biopsy is usually not needed. Seek consultation if needed.

2. Define the pathologic as well as the clinical activity of the gut inflammatory disease.

3. Search for other EIMs, particularly eye and bone EIMs.

4. Aggressively treat the gut inflammation as the EN will follow the course to resolution.

5. Recurrent EN likely requires reassessment of the IBD status and management as well as dermatologic consultation.

\section{Pyoderma Gangrenosum}

Pyoderma gangrenosum (PG) is the second most common (0.5$4.9 \%$ ) dermatologic EIM in patients with IBD, is perhaps more common in females and in UC $(8.6 \%)$ compared to CD $(3.6 \%)$, and is by far the most serious of the cutaneous EIMs. ${ }^{17}$ The relationship of PG to IBD disease activity is unpredictable as is the response to any specific therapy. ${ }^{18}$ While corticosteroids and antiTNF- $\alpha$ treatment certainly work, a favorable response here is not a given. PG morbidity frequently requires hospitalization, can be debilitating, and adversely affect quality of life. ${ }^{9,12,17}$ PG is characterized as a severe, sterile, neutrophilic dermatosis. Clinical categories of PG include ulcerative, pustular, bullous, and vegetan forms. ${ }^{9}$ It most often affects body parts subject to minor trauma such as the lower extremities or near abdominal ostomy sites. PG often, but not universally, begins with a minor trauma such as a pin prick or bump evolving sequentially, oftentimes rapidly to pustule, focal necrosis of the dermis, to deep, very painful, reddish-violaceous bordered ulceration. Excellent images of typical PG can be found in the literature. ${ }^{9,17}$ This phenomenon of an exaggerated inflammatory response to cutaneous trauma is referred to as "pathergy" and plays an important role in accurately defining and managing PG. Therefore, in PG it is critically important to avoid ulcer debridement as this traumatic intervention may trigger ulcer progression. As depicted in the Figure 1 cartoon, the trauma of pathergy perhaps triggers the proinflammatory cascade that promotes the exacerbation of PG. Dermatology consultation is essential for diagnostic accuracy and management. In $20 \%$ of cases, PG is diagnosed in error. ${ }^{17}$ It is important to rule out leg ulcers due to venous insufficiency, malignancy, insect bites, autoimmune disease, vasculitis, ischemia, Sweet's syndrome (discussed below), infection, and to differentiate primary bacterial infection from associated, but not causative superinfection, particularly Staphylococcus aureus. The management of PG has no gold standard. Small ulcers may be managed with topical steroid or tacrolimus ointment, cyclosporine solutions, antibiotics, and careful application of compression bandages to reduce swelling. Larger ulcers are best managed with systemic therapy, preferably as an in-patient. Corticosteroids and antiTNF- $\alpha$ biologics are the mainstay of management in this situation. In all case expert pain management, avoidance of superinfection, and superb wound care are essential.

The management decisions that are paramount for PG include:

1. Dermatological Consultation; biopsy is not essential if dermatologic diagnostic criteria present.

2. Culture wound for superinfection. Provide antibiotics initially and be guided by culture results.

3. Define and manage the gut inflammation.

4. Consider the differential diagnosis vigorously.

5. Establish an expert program of wound care.

6. Determine the disposition for care, outpatient for small ulcers $v s$. hospitalization for advanced ulcers, multiple ulcers, and need for systemic therapy with steroids, or one of several biologics.

7. If improvement does not occur with 2-4-weeks, be prepared to change the treatment program.

\section{Metastatic Crohn's Disease}

Although the nomenclature is somewhat distracting, metastatic crohn's disease (MCD) refers to the noncaseating granulomas with multinucleated giant cells in the dermis upon skin lesion biopsy and which are a hallmark, albeit not universally found, of intestinal pathology of CD. ${ }^{9}$ Although rare, MCD presents in an indolent fashion as subcutaneous nodules or non-healing ulcers of the face, lower extremities, or the genitals ( $50 \%$ of cases vulvar or testicular). Images of MCD of the face may be found in the literature. ${ }^{18}$ The lesions are painful and pruritic. MCD does not appear to follow the activity of the intestinal disease, may occur in children or adults, and has a variable response to steroids or anti-TNF- $\alpha$ agents. ${ }^{18}$ If all else fails, surgical management may be required. Dermatology consultation is in order. 


\section{Sweet's Syndrome}

Sweet's syndrome (SS), first described by Robert Douglas Sweet in 1964 is also known as acute febrile neutrophilic dermatosis. It is a rare $(<1 \%$ of IBD patients) pathology, is more often seen in adult females with active IBD (UC>CD, 9). Clinically, SS is characterized by the sudden onset of fever, headache, fatigue, an elevated white blood cell count, and tender, red, well-demarcated papules and plaques that show dense infiltrates by neutrophil granulocytes on histologic examination. ${ }^{8,9}$ Topical or systemic steroids for two to four weeks and good control of the IBD have been successful treatments. The use of anti-TNF- $\alpha$ has been reported in the literature but the data are sparse. SS has also been reported as a paradoxical reaction to anti-TNF- $\alpha$ and multiple other drugs. ${ }^{19}$

\section{Inflammatory and Non-inflammatory Arthropathies of Inflammatory Bowel Diseases}

Musculoskeletal symptoms are described in $6-46 \%$ of IBD. This wide variation in the medical literature is due to both how the data was collected and probably also to less than facile understanding of the rheumatological definitions of various arthropathies. All this notwithstanding, joint pains are considered the most common $^{11}$ of the IBD EIMs, and occur more often in CD (33\%) than UC (21\%). To answer clinical questions about a painful joint we must consult our rheumatology colleagues for a few definitions. ${ }^{20}$

Spondyloarthropathies: This is a group of inflammatory arthropathies which are seronegative, i.e. negative rheumatoid factor, and share distinct clinical, radiologic, and genetic features. This group includes ankylosing spondylitis, Reiter's syndrome, juvenile spondyloarthropathy, psoriatic arthritis, and enteropathic arthritis of inflammatory bowel disease (IBD-SpA). This last listed pathology is the concern of this communication.

Spondyloarthritis of Inflammatory Bowel Diseases: This group of inflammatory bone diseases includes an axial group of ankylosing spondylitis (AS) and sacroiliitis (SI) and a peripherial group of Type 1 (also called pauciarticular) and Type 2 (also called polyarticular). The AS of IBD is not exactly the same disorder as idiopathic AS. Genetically, HLA-B27 is present in nearly all cases (it is a criterion for diagnosis) of idiopathic AS but occurs in only a minority of IBD-associated AS. Hence, HLA-B27 is not particularly helpful as a diagnostic tool in IBD. However, HLA-B27 positive IBD patients are at increased risk for AS.,

Non-inflammatory arthritis: In IBD, this is a diagnosis of exclusion and most often called "arthralgia". It would include degerative joint disease or fibromyalgia complaints or joint discomfort associated with physically demanding occupation. There is also the "suspicious arthralgia". This diagnosis is made when the clinician has a highented sense of awareness or crystal ball predicting that the non-inflammatory appearing but painful joints will evolve with erythema and swelling to qualify as an inflammatory arthritis. Indeed, arthalgias do favorably respond to anti-TNF- $\alpha$ biologics as do most spondyloarthritis of inflammatory bowel diseases (IBD$\mathrm{SpA})$.

With disciplined attention (Table 3) to the rheumatological definitions of the IBD-SpA classes, rheumatology consultation, and management of the gut components of IBD, most patients can be managed successfully. It is important to define AS as early as possible, not only for protection of quality of life but also because AS is a progressive disorder.

The prinicpal considerations and decisions to be made

\begin{tabular}{|c|c|c|c|c|}
\hline Type & Frequency & Clinical Findings & First Line Therapy & Second Line Therapy \\
\hline \multicolumn{5}{|l|}{ Axial SpA } \\
\hline AS & $2-4 \%$ & $\begin{array}{l}\text { Prevalence CD>>UC; } \\
\text { AM back stiffness which decrease } \\
\text { with activity; } \\
\text { Pain<age } 45 \text { yrs, >3 mos; } \\
\text { Spine films/MRI for dx; } \\
\text { May occur prior to IBD dx; }\end{array}$ & $\begin{array}{l}\text { rheumatology consult } \\
\text { COX-2 inhibitors } \\
\text { Physical therapy }\end{array}$ & $\begin{array}{l}\text { anti-TNF- } \alpha \text { agents } \\
\text { corticosteroids }\end{array}$ \\
\hline SI & $10 \%$ & $\begin{array}{l}\text { HLA-B27 usually negative; } \\
\text { Buttocks pain; } \\
\text { Usually does not progress to AS } \\
\text { spine films/MRI for } \mathrm{dx} \text {; }\end{array}$ & \multicolumn{2}{|c|}{ Same as for AS } \\
\hline $\begin{array}{l}\text { Peripheral SpA type I } \\
\text { (pauciarticular) }\end{array}$ & $2-6 \%$ & $\begin{array}{l}\text { prevalence } C D>\cup C<5 \text { large joints; } \\
\text { asymmetrical; } \\
\text { Self-limited (<10week); } \\
\text { Associated with IBD flare; } \\
\text { Associated with EN, uveitis. }\end{array}$ & $\begin{array}{l}\text { manage GI disease } \\
\text { COX-2 inhibitors } \\
\text { rheumatology consult }\end{array}$ & $\begin{array}{l}\text { sulfasalazine anti-TNF- } \alpha \\
\text { agents corticosteroids }\end{array}$ \\
\hline $\begin{array}{l}\text { Type } 2 \\
\text { (polyarticular) }\end{array}$ & & $\begin{array}{l}>5 \text { small joints; } \\
\text { Symmetrical; } \\
\text { Associate with uveitis persistent } \\
\text { symptoms Independent of IBD }\end{array}$ & \multicolumn{2}{|c|}{ Same as for type I } \\
\hline $\begin{array}{l}\text { Abbreviations: } C D-C r o h n \\
\text { cyclo-oxygenase inhibitor }\end{array}$ & $\begin{array}{l}\text { sease; UC-ulc } \\
\text { 2;Anti-TNF }\end{array}$ & $\begin{array}{l}\text { tive colitis; SpA-spondyloarthritis; } A \text { S-anky } \\
\text { anti-tumor necrosis factor alpha; } E N \text {-ery }\end{array}$ & $\begin{array}{l}\text { osing spondylitis; SI- sacroi } \\
\text { hema nodosum. }\end{array}$ & liitis; COX-2 \\
\hline
\end{tabular}


patients, runs an independent course, and may precede the diagnosis of IBD in a substantial proportion of cases. ${ }^{16}$ Scleritis, the least common of the three, also runs a course independent of the intestinal disease activity and may occur antecedent to the diagnosis of IBD.

There are three challenges, questions, and/or decisions for the clinician:

1. What is the nature of the eye disorder, i.e. conjunctivitis, episcleritis, scleritis, uveitis?

2. Is the disorder an EIM of IBD or due to a separate event, i.e. prior eye surgery, trauma, viral infection?

3. What is the best course of care? Table 3 provides assistance with the challenges of diagnosis and management.

From Table 4, isolated, transient, ocular redness, and the absence of pain, photophobia, or changes in visual acuity all suggests a benign course. The presence of these signs and symptoms requires support from ophthalmology consultants to ensure a favorable outcome.

\section{Hepatopancreaticobiliary Extraintestinal Manifestations of Inflammatory Bowel Diseases}

The IBD patient often presents with abnormal liver function studies. Hence this is the most common (perhaps 30\%) IBD EIM when all categories (Table 1) are considered. ${ }^{6}$ The most serious is primary sclerosing cholangitis with prevalence of $4-5 \%$ in the IBD population 6,11 ; the most common is non-alcoholic fatty liver disease (NAFLD, 23\%) which is in the range for the general public. Other hepatopancreaticobiliary EIMs that occur often enough to also require constant consideration include drug-induced liver injury, hepatic and portal vein thrombosis, autoimmune liver disease, and acute pancreatitis of multiple etiologies. Primary sclerosing cholangitis, a "true" EIM, is a constant challenge for the IBD clinician. Its clinical pattern, associated cancers, and decision-making tasks are discussed below.

\section{Primary Sclerosing Cholangitis}

Primary sclerosing cholangitis (PSC) is a chronic, progressive, in$2-5 \%$ of IBD patients. It runs a course in parallel with intestinal activity. Uveitis, the most dangerous, occurs in up to $3 \%$ of IBD

\begin{tabular}{|c|c|c|}
\hline Disorder & Clinical Characteristics & Management \\
\hline Episcleritis & $\begin{array}{l}\text { Eye redness, some irritation, but pain is absent } \\
\text { and vision not threatened; } \\
\text { Transient disorder; } \\
\text { May parallel disease activity }\end{array}$ & $\begin{array}{l}\text { Manage the gut activity; } \\
\text { Topical steroids may help; } \\
\text { Watchful waiting may suffice; }\end{array}$ \\
\hline Scleritis & $\begin{array}{l}\text { Pink or violet sclera, pain is characteristic and } \\
\text { may be severe; } \\
\text { Vision may be impaired; } \\
\text { Rule out varicella zoster and herpes simplex }\end{array}$ & $\begin{array}{l}\text { Consult ophthalmology urgently; } \\
\text { Systemic oral NSAIDS or steroids; } \\
\text { TNF- } \alpha \text { inhibitor for severe cases } \\
\text { manage the gut activity }\end{array}$ \\
\hline Uveitis & $\begin{array}{l}\text { Acute or subacute, very painful, vision at high-risk; } \\
\text { Photophobia, headaches, facial pain; } \\
\text { May be associated with other EIMs; } \\
\text { More common in CD (II.I\%) vs. UC ( } 5.6 \%) \text {; } \\
\text { Family history of IBD }\end{array}$ & $\begin{array}{l}\text { Consult ophthalmology urgently; } \\
\text { As above for scleritis; } \\
\text { Manage the gut activity }\end{array}$ \\
\hline
\end{tabular}


flammatory, cholestatic, hepatobiliary pathology possessing a predisposition for inflammatory bowel disease. PSC has a UC (4\% vs. $1 \%$ in $\mathrm{CD}$ ) and male predilections; and may precede the diagnosis of IBD in $14.3 \%$ of IBD patients. ${ }^{6,11,16}$ It is theorized to develop through a combination of genetic and immunologic factors resulting in adverse targeting of the biliary system by activated lymphocytes and autoantibodies. The pathogenesis may or may not be influenced by the activity of the underlying IBD which is almost always colonic in location. The hepatic inflammatory consequences include large and small intra-and extra-hepatic bile duct strictures, cholestasis, leading to cirrhosis, hepatic failure, and/or cholangiocarcinoma ${ }^{25}$ PSC has no known effective, course altering, medical treatment; steroids, ursodeoxycholic acid, and biologics do not improve survival. Only liver transplantation offers the PSC patient a survival benefit. PSC may recur in $25 \%$ of successfully transplanted patients. ${ }^{26,27}$ Cholangiocarcinoma in IBD is an ageand disease- duration dependent cancer requiring surveillance to define the disease at the earliest possible time if and survival is to expected. ${ }^{26,27}$ PSC is associated with an increased risk of colon cancer for which surveillance screening colonoscopy every 1-2-years is recommended in patients with $\mathrm{IBD} .^{27}$ In addition to cholangiocarcinoma and colon cancer, PSC carries also additional risks for gallbladder and pancreatic cancer. In PSC the liver function tests are persistently abnormal. Gallbladder disease, NAFLD, alcoholic liver disease, and drug-induced liver disease are much more prevalent parts of the differential diagnosis. Imaging studies with ultrasound and magnetic resonance cholangiopancreatography (MRCP) can be diagnostic. However, in 10\% of PSC cases, imaging may be nor$\mathrm{mal}$, in which case consideration for liver biopsy is in order to diagnose viral, autoimmune, drug-induced liver disease, primary biliary cirrhosis, or small duct PSC ${ }^{28}$; biopsy results will provide direction for management decisions. Be mindful that the intrahepatic pathology of PSC may be patchy and sampling error is always a risk. In the IBD patient, small duct PSC is a variant of IBD-PSC as the latter generally requires both large and small duct pathology. Small duct PSC has been called pericholangitis and may require differentiation from autoimmune hepatitis. If both PSC and autoimmune hepatitis features are present, it may qualify as overlap syndrome (also known as "PSC with autoimmune features." 28 Small duct PSC has a more favorable prognosis than classical PSC. It does not progress to cholangiocarcinoma unless it first progresses (12-20\%) to large duct PSC. ${ }^{28}$ Secondary sclerosing cholangitis, most commonly due to chronic biliary obstruction, bile duct trauma, chemotherapy, or ischemia can usually be separated from PSC with an adequate history. ${ }^{29}$

The following decision-making considerations are appropriate for the diagnosis and care of patients with PSC:

1. Many patients are asymptomatic early in the course of PSC. However, pruritis is common. Antihistamines, cholestyramine, rifampicin, opioids, and bezafibrate (available in Canada) are among current choices for symptomatic relief.

2. Obtain alkaline phosphatase with other liver function tests, CA19-9, CBC, C-reactive protein.

3. Consultation with hepatologist.
4. Evaluate the status of IBD; fecal calprotectin, surveillance colonoscopy if not done in past year.

5. Obtain ultrasound of the liver, gallbladder, and pancreas.

6. Proceed with MRCP which is the imaging study of choice for PSC.

7. If MRCP negative, consider other diseases including small duct PSC and proceed with liver biopsy.

\section{DRUG-INDUCED LIVER INJURY IN INFLAMMATORY BOWEL DISEASES}

IBD is a chronic disease with an ever-increasing list of medications being made available. Fortunately, the newer the therapeutic agent, the less likely the liver will be assaulted. While 5-aminosalicylates (rare), thiopurines (3\%), and cyclosporine $(32 \%)$ are associated with risks of hepatotoxicity ${ }^{30,31}$ and methotrexate a dose-dependent risk of hepatic fibrosis, ${ }^{32}$ the biologics are only rarely associated with hepatotoxicity. As a matter of prudence and according to package inserts, monitoring of liver function tests perhaps semiannually is recommended for all IBD patients whether on biologics or not. For the biologics, the risk is in reactivating hepatitis B (but not C). There appears to be no substantial risk of hepatotoxicity with vedolizumab, ustekinumab, or tofacitinib.

\section{PARADOXICAL DERMATOLOGIC AND ARTHROPATHIC REACTIONS TO TNF- $\alpha$ INHIBITORS -}

The term "paradoxical" has entered the lexicon of IBD because skin and bone diseases, such as psoriasiform eczema and IBD-SpA that are typically treated with anti-TNF- $\alpha$ biologics, may arise while the patient is being treated with that same biologic for the gut component of IBD. ${ }^{33-35}$ Typically, the gut may be in clinical remission. In IBD patients, the psoriasiform lesions are notably somewhat different than the lesions of classical psoriasis. Skin lesions may arise at any time during the course of anti-TNF- $\alpha$ exposure. The most common lesion is psoriasiform eczema (30.6\%), occurs at flexures, genitalia, or scalp. The cartoon of Figure 1. provides some basis for understanding why psoriasis is more common in IBD and how the skin disease may arise even while under treatment with a biologic which is effective in the management of psoriasis. Risk factors seem to include smoking, CD, obesity, and female gender while thiopurines, methotrexate and UC may be protective. With dermatologic consultation, most cases can be managed with topical therapies or systemic steroids without discontinuation of the biologic. Recurrences are common and it may be clinically prudent to discontinue the anti-TNF- $\alpha$ therapy perhaps in $10 \%$ of cases. In this situation, ustekinumab may be an excellent alterative treatment for the gut component with also attendant resolution of the skin disease. ${ }^{36}$ Recalling, the central roles of IL-23 and TNF- $\alpha$ in the propagation of the IMIDs, the balance, and inter-dependence of the proinflammatory cytokines, the clinician may better understand, albeit with incomplete scientific explanation, these paradoxical disorders and the approach to management. They are only "paradoxical" because we do not fully understand the balance and interplay of the cytokines with our perhaps brutish, but current, use of the biologics in the treatment of IBD. 
Theibault et $\mathrm{al}^{35}$ reported that paradoxical arthropathy of anti-TNF- $\alpha$ therapy may occur in up to $11 \%$ of IBD patients, most often in long-standing CD in clinical remission. In their prospective, single-center study, rheumatologic consultation was offered to all IBD patients being maintained on infliximab. In the one-year study, of $65 \mathrm{CD}$ and $15 \mathrm{UC}$ patients, 9 (11\%) patients, all CD, were diagnosed with paradoxical arthropathy. Of note, the IBD itself was considered to be the cause of a new arthropathy EIM in an equal number of cases. Most likely the CD was active pathologically if not clinically! In the paradoxical cases, it was not necessary to discontinue the biologic; methotrexate was beneficial in two of three patients who received this appended therapy. As with all the EIMs, this study demonstrates the value of subspecialty consultation.

Decision-making for paradoxical disease:

1. Rheumatology or dermatology consultation.

2. Determine the pathologic status of gut activity in addition to clinical activity.

3. Manage the paradoxical disease as recommended.

4. Continue the current biologic in most cases.

5. Manage EIMs, paradoxical reactions, and the gut components of IBD with equal vigor.

\section{CONCLUSION}

Inflammatory bowel diseases is in the class of immunologicallymediated inflammatory diseases and EIMs reflect the systemic nature of IBD. The EIMs of IBD and many IBD-associated diseases perhaps share in common immunopathologic and cytokine-mediated pathways. Diagnosis and management of the EIMs depend upon an understanding of these pathways, multi-specialty clinical involvement, and the selective application of therapeutics taken from full armamentarium of treatments available for respective EIM targets. Application of the specific decision-making processes and considerations presented will promote accurate diagnoses and effective therapeutic interventions.

\section{REFERENCES}

1. Guan Q. A comprehensive review and update on the pathogenesis of inflammatory bowel disease. J Immunol Res. 2019; 2019: 7247238. doi: $10.1155 / 2019 / 7247238$

2. Strober W, Fuss IJ. Pro-inflammatory cytokines in the pathogenesis of IBD. Gastroenterology. 2011; 140: 1756-1767. doi: 10.1053/j. gastro.2011.02.016

3. Chang, JT. Pathophysiology of inflammatory bowel diseases. $N$ Engl J Med. 2020; 383: 2652-2664. doi: 10.1056/NEJMra 2002697

4. Guillo L, D’Amico, Serrero M, Angioi K, Loeuille D, Costanzo A, et al. Assessment of extraintestinal manifestations in inflammatory bowel diseases: A systematic review and a proposed guide for clinical trials. United European Gastroenterol J. 2020; 8(9): 1013-1030. doi: $10.1177 / 2050640620950093$

5. Juillerat P, Manz M, Sauter B, Zeitz J, Vavricka SR, IBDnet S, et al. Therapies in inflammatory bowel disease patients with extraintestinal manifestations. Digestion. 2020; 101 (suppl 1): 83-97. doi: $10.1159 / 000502816$

6. Harbord M, Annese V, Vavricka SR, Allez M, Acosta MB, Boberg KM, et al. The first European evidenced-based consensus on extra-intestinal manifestations in inflammatory bowel disease. J Crohn's and Colitis. 2016; 10(3): 239-254. doi: 10.1093/ecco-jcc/ jjv213

7. Hedin CRH, Vavricka SR, Stagg AJ, Schoepfer A, Raine T, Puig $\mathrm{L}$, et al. The pathogenesis of extraintestinal manifestations: Implications for IBD research, diagnosis, and therapy. J Crobn's and Colitis. 2019; 13(5): 541-554. doi: 10.1093/ecco-jcc/jjy191

8. Garber A, Regueiro M. Extraintestinal manifestations of inflammatory bowel disease: Epidemiology, and management. Curr Gastro Rep. 2019; 21: 31-44. doi: 10.1007/s11894-019-0698-1

9. Huang BL, Chandra S, Shin DQ. Skin manifestations of inflammatory bowel disease. Front. Physiol. 2012; 3: 1-13. doi: 10.3389/ fphy.2012.00013

10. Bunted K, Beaker T. The TH 17 cells and the IL-23/IL-17 axis in the pathogenesis of periodontitis and immune-mediated inflammatory diseases. Int J Mol Sci. 2019; 20: 3394-3418. doi: 10.3390/ ijms20143394

11. Vavricka SR, Brun L, Ballabeni P, Pittet V, Vavricka BMP, Zeitz $\mathrm{J}$, et al. Frequency and risk factors for extraintestinal manifestations in the Swiss inflammatory bowel disease cohort. Am J Gastroenterol. 2011; 106: 110-119. doi: 10.1038/ajg.2010.343

12. Roth N, Biedermann L, Fournier N, Butter M, Vavricka SR, Navarini AA, et al. Occurrence of skin manifestations in patients of the Swiss inflammatory bowel disease cohort study. PLoS One. 2019; 14(1): e0210436. doi: 10.1371/journal.pone.0210436

13. Modigliani R, Mary JY, Simon JF, Cortot A, Soule JC, Gendre $\mathrm{JP}$, et al. Clinical, biological, and endoscopic picture of attacks of Crohn's disease. evolution on prednisolone. Group d'Etude therapeutique des affections inflammatoires digestives. Gastroenterology. 1990; 98(4): 811-818. doi: 10.1016/0016-5085(90)90002-i

14. Sipponen T, Nuutinen H, Turunen U, Färkkilä M. Endoscopic evaluation of Crohn's disease activity: Comparison of the CDEIS and the SES-CD. Inflamm Bowel Dis. 2010; 16(12): 2131-2136. doi: 10.1002/ibd. 21300

15. Vavricka SR, Gubler M, Gantenbein C, Spoerri M, Froehlich F, Seibold F, et al. Anti-TNF treatment of extraintestinal manifestations of inflammatory bowel disease in the Swiss cohort 
study. Inflamm Bowel Dis. 2017; 23(7): 1174-1181. doi: 10.1097/ MIB.0000000000001109

16. Vavricka SR, Rogler G, Gantenbein C, Spoerri M, Vavricka MP, Navarini AA, et al. Chronological order of appearance of extraintestinal manifestations relative to time of diagnosis of IBD diagnosis in the Swiss inflammatory bowel disease cohort. Inflamm Bowel Dis. 2015; 21(8): 1794-1800. doi: 10.1097/MIB.0000000000000429

17. Weizman AV, Huang B, Targan S, Dubinsky M, Fleshner P, Kaur M, et al. Pyoderma gangrenosum among patients with inflammatory bowel disease: A descriptive cohort study. J Cutan Med Surg. 2015; 19(2): 125-131. doi: 10.2310/7750.2014.14053

18. Aberumand B, Howard J, Howard J. Metastatic Crohn's disease: An approach to an uncommon but important cutaneous disorder. Biomed Res Int. 2017; 2017: 8192150. doi: 10.1155/2017/8192150

19. Garcovich S, De Simone C, Genovese G, Berti E, Cugno M, Marzano AV. Paradoxical skin reactions to biologics in patients with rheumatologic disorders. Front Pharmacol. 2019; 10: 282-295. doi: 10.3389/fphar.2019.00282

20. Rudwalet M, van der Heijde D, Landewe R, et al. The assessment of spondyloarthritis international society classification criteria for peripheral spondyloarthritis and for spondyloarthritis in general. Ann Rheum Dis. 2011; 70(1): 25-31. doi: 10.1136/ard.2010.133645

21. Bourikas LA, Papadakis KA. Musculoskeletal manifestations of inflammatory bowel disease. Inflamm Bowel Dis. 2009; 15(12): 1915-1924. doi: 10.1002/ibd.20942

22. Biedermann L, Renz L, Fournier N, Rossel J-B, Butter M, Bluemel S, et al. Uveitis manifestations in patients of the Swiss inflammatory bowel disease cohort study. Ther Adv Gastroenterol. 2019; 12: 1756284819865142. doi: 10.1177/1756284819865142

23. Salmon JF, Wright JP, Murray ADN. Ocular manifestations in Crohn's disease. Ophthalmology. 1991; 98(4): 480-484. doi: 10.1016/ SO161-6420(91)32268-1

24. Mintz R, Feller ER, Bahr RL, Shah SA. Ocular manifestations of inflammatory bowel disease. Inflamm Bowel Dis. 2004; 10(2): 135139. doi: 10.1097/00054725-200403000-00012

25. Navaneethan U, Shen B. Hepatopancreaticobiliary manifestations and complications associated with inflammatory bowel disease. Inflamm Bowel Dis. 2010; 16(9): 1598-1619. doi: 10.1002/ ibd.21219

26. Weismuller T, Trivedi P, Bergquist A, Imam M, Lenzen H, Ponsioen CY, et al. Patient age, sex, and inflammatory bowel disease phenotype associated with the course of primary sclerosing chol- angitis. Gastroenterology. 2017; 152(8): 1975-1984.e8. doi: 10.1053/j. gastro.2017.02.038

27. Chapman R, Fevery J, Kalloo A, Nagorney DM, Boberg KM, Shneider B, et al. Diagnosis and management of primary sclerosing cholangitis. Hepatology. 2010; 51(2): 660-678. doi: 10.1002/ hep. 23294

28. Bjornsson E, Olsson R, Bergquist A, Lindgren S, Braden B, Chapman RW, et al. The natural history of small-duct primary sclerosing cholangitis. Gastroenterology. 2008; 134(4): 975-980. doi: 10.1053/j.gastro.2008.01.042

29. Ruemmele P, Hofstaedter F, Gelbmann CM. Secondary sclerosing cholangitis. Nat Rev Gastroenterol Hepatol. 2009; 6(5): $287-$ 295. doi: $10.1038 /$ nrgastro.2009.26

30. Moran GW, Dubeau M-F, Kaplan GG, Yang H, Eksteen B, Ghosh S, et al. Clinical predictors of thiopurine-related adverse events in Crohn's disease. World J Gastroenterol. 2015; 21(25): 77957804. doi: $10.3748 /$ wjg.v21.i25.7795

31. Kassianides C, Nussenblatt R, Palestine AG, Mellow SD, Hoofnagle JH, et al. Liver injury from cyclosporine A. Dig Dis Sci. 1990; 35(6): 693-697. doi: 10.1007/BF01540169

32. Bath RK, Brar NK, Forouhar FA, Wu GY. A review of methotrexate-associated hepatotoxicity. J Dig Dis. 2014; 15(10): 517-524. doi: $10.1111 / 1751-2980.12184$

33. Cleynen I, Van Moerkercke W, Billiet T, Vandecandelaere P, Casteele NV, Breynaert C, et al. Characteristics of skin lesions associated with ant-tumor necrosis factor therapy in patients with inflammatory bowel disease: A cohort study. Ann Intern Med. 2016; 164(1): 10-22. doi: 10.7326/M15-0729

34. Melo F, Magina S. Clinical management of anti-tnf-alphainduced psoriasis or psoriasiform lesions in inflammatory bowel disease patients: A systematic review. Int J Dermatol. 2018; 57(12): 1521-1532. doi: $10.1111 /$ ijd.14072

35. Thibault H, Thibault P, Guingona C, Guillaume N, Wacrenier A, Sabbagh C, et al. Paradoxical articular manifestations in patients with inflammatory bowel diseases treated with infliximab. Eur J Gastroenterol Hepatol. 2016; 28(8): 876-1881. doi: 10.1097/ MEG.0000000000000643

36. Ezzedine K, Visseaux L, Cadiott G, Brixi H, Bernard P, Reguiai $\mathrm{Z}$, et al. Ustekinumab for skin reactions associated with anti-tumor necrosis- $\alpha$ agents in patients with inflammatory bowel diseases: A single-center retrospective study. J Dermatol. 2019; 46(4): 322-327. doi: $10.1111 / 1346-8138.148$ 